\title{
Inhibitor of Endocannabinoid Deactivation Protects Against In Vitro and In Vivo Neurotoxic Effects of Par...
}

Article in Journal of Molecular Neuroscience · August 2017

DOI: $10.1007 /$ s12031-017-0963-4

CITATIONS

0

11 authors, including:

Karen L G Farizatto

University of North Carolina at Pembroke

9 PUBLICATIONS 30 CITATIONS

SEE PROFILE

\section{Spyros P Nikas}

Northeastern University

53 PUBLICATIONS 767 CITATIONS

SEE PROFILE
READS

47

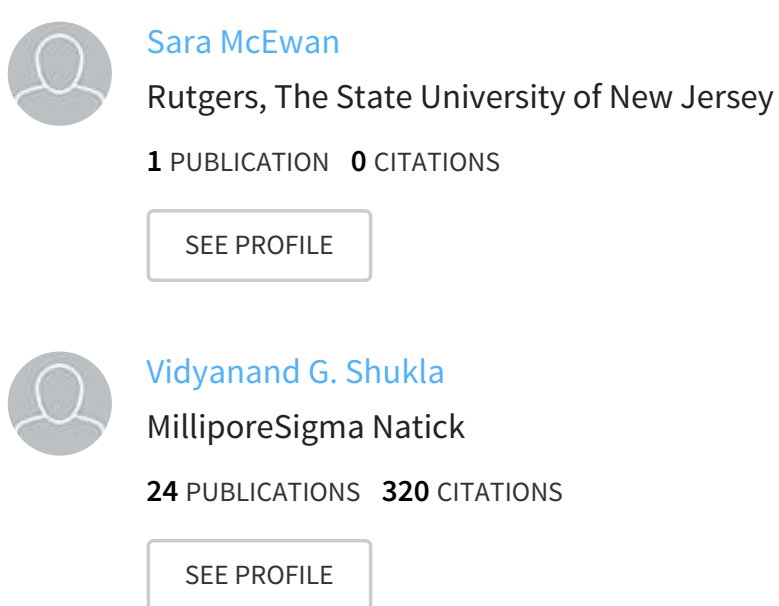

Some of the authors of this publication are also working on these related projects:

synthesis of cannabinoid View project 


\title{
Inhibitor of Endocannabinoid Deactivation Protects Against In Vitro and In Vivo Neurotoxic Effects of Paraoxon
}

\author{
Karen L. G. Farizatto ${ }^{1}$ - Sara A. McEwan ${ }^{1,2} \cdot$ Vinogran Naidoo $^{1,3} \cdot$ Spyros P. Nikas $^{4}$. \\ Vidyanand G. Shukla ${ }^{4} \cdot$ Michael F. Almeida ${ }^{1} \cdot$ Aaron Byrd $^{1} \cdot$ Heather Romine ${ }^{1}$. \\ David A. Karanian ${ }^{5}$. Alexandros Makriyannis ${ }^{4}$ Ben A. Bahr ${ }^{1,5}$
}

Received: 3 May 2017 / Accepted: 2 August 2017

(C) Springer Science+Business Media, LLC 2017

\begin{abstract}
The anticholinesterase paraoxon (Pxn) is related to military nerve agents that increase acetylcholine levels, trigger seizures, and cause excitotoxic damage in the brain. In rat hippocampal slice cultures, high-dose Pxn was applied resulting in a presynaptic vulnerability evidenced by a $64 \%$ reduction in synapsin IIb (syn IIb) levels, whereas the postsynaptic protein GluR1 was unchanged. Other signs of Pxninduced cytotoxicity include the oxidative stress-related production of stable 4-hydroxynonenal (4-HNE)-protein adducts. Next, the Pxn toxicity was tested for protective effects by the fatty acid amide hydrolase (FAAH) inhibitor AM5206, a compound linked to enhanced repair signaling through the endocannabinoid pathway. The Pxn-mediated declines in syn IIb and synaptophysin were prevented by AM5206 in the slice cultures. To test if the protective results in the slice model translate to an in vivo model, AM5206 was injected i.p. into rats, followed immediately by subcutaneous Pxn administration. The toxin caused a pathogenic cascade initiated by seizure events, leading to presynaptic marker decline and
\end{abstract}

Karen L. G. Farizatto and Sara A. McEwan contributed equally to this study.

Ben A. Bahr

bahr@uncp.edu

1 Biotechnology Research and Training Center, University of North Carolina-Pembroke, Pembroke, NC 28372-1510, USA

2 Present address: Robert Wood Johnson Medical School, Rutgers University, New Brunswick, NJ, USA

3 Present address: Department of Human Biology, University of Cape Town, Cape Town, South Africa

4 Center for Drug Discovery, Northeastern University, Boston, MA, USA

5 Neurosciences Program, University of Connecticut, Storrs, CT, USA oxidative changes in the hippocampus and frontal cortex. AM5206 exhibited protective effects including the reduction of seizure severity by $86 \%$, and improving balance and coordination measured $24 \mathrm{~h}$ post-insult. As observed in hippocampal slices, the FAAH inhibitor also prevented the Pxn-induced loss of syn IIb in vivo. In addition, the AM5206 compound reduced the 4-HNE modifications of proteins and the $\beta 1$ integrin activation events both in vitro and in vivo. These results indicate that Pxn exposure produces oxidative and synaptic toxicity that leads to the behavioral deficits manifested by the neurotoxin. In contrast, the presence of FAAH inhibitor AM5206 offsets the pathogenic cascade elicited by the Pxn anticholinesterase.

Keywords Paraoxon - Anticholinesterase $\cdot$ Synaptic decline . AM5206 $\cdot$ Neuroprotection $\cdot$ Excitotoxicity

\section{Introduction}

Increased activation of acetylcholine receptors has been associated with excitotoxic damage in the central nervous system (Prager et al. 2015). Neurotoxic agents such as paraoxon (Pxn) and chlorpyrifos, one of the most widely used pesticides in the world (Reynolds 2017), inhibit the enzyme acetylcholinesterase which leads to an accumulation of acetylcholine in synapses and subsequent overstimulation of cholinergic receptors. As a result, the cholinergic crisis triggers seizures, long-term behavioral changes, and reduces cognition (see Sánchez-Santed et al. 2004; Prager et al. 2015). The important issue of toxicity in humans and animals is due to the organophosphates and their metabolites having relatively low level of surveillance system regarding their use.

The danger of anticholinesterase toxins obviously includes the occurrence of lethality. Asymptomatic low-level 
exposures can also leave the brain vulnerable to subsequent brain insults such as traumatic injury, stroke events, and seizures (see Munirathinam and Bahr 2004). This reinforces the idea that certain neurotoxins like Pxn have actions beyond anticholinesterase effects, for example, other neurotransmitter systems may be involved in the propagation of the brain damage. Note, Pxn increases the release of glutamate in the hippocampus (Kozhemyakin et al. 2010). Whereas, GABA uptake was significantly reduced in both cerebral cortex and hippocampus of Pxn-treated rats (Mohammadi et al. 2008). In addition, the consequences of Pxn exposure can cause neurological problems later in life, or disrupt developmental processes underlying synaptic connectivity and cognitive ability in children which appear to be particularly vulnerable to anticholinesterase effects (Rotenberg and Newmark 2003). Thus, Pxn insults appear to be linked with different alterations that disturb cellular homeostasis.

Cannabinergic mechanisms of neuroprotection represent a plausible strategy for neuronal maintenance and/or repair in order to offset damage caused by toxin exposure and excitotoxic insults (Fernandez-Ruiz et al. 2010; Naidoo et al. 2011). The endocannabinoid system has been linked to protective on-demand responses in the brain. It works against seizure damage, stroke/ischemia, traumatic brain injury, and multiple sclerosis (Bahr et al. 2006; Fernandez-Ruiz et al. 2010; Hwang et al. 2010; Sagar et al. 2010; Rosenberg et al. 2017). Endocannabinoid regulation occurs through the inhibition of fatty acid amide hydrolase (FAAH) and monoacylglycerol lipase (MAGL), resulting in enhancing effects on anandamide (AEA) and 2-arachidonoyl glycerol (2-AG), respectively.

Targeting the endocannabinoid-degrading enzyme FAAH may be an ideal strategy to promote endogenous repair pathways to act against negative effects caused by Pxn. Here, we utilized a member of the FAAH inhibitor group of agents that have been developed, compounds that are known to increase endogenous levels of AEA through FAAH inactivation (Kathuria et al. 2003; Karanian et al. 2007). We tested whether a reversible FAAH inhibitor, the trifluoromethyl ketone ana$\log$ AM5206, prevents pathogenic events triggered by the anticholinesterase Pxn, focusing mainly in the hippocampus, a brain region that exhibits a vulnerability to different types of neuropathogeneses.

\section{Materials and Methods}

Sprague-Dawley rat litters and young Sprague-Dawley male rats were obtained from Charles River Laboratories (Wilmington, MA) and housed in accordance with guidelines from the National Institutes of Health. Brain tissue from 12day-old rats was rapidly removed to prepare hippocampal slices as described previously (Bendiske et al. 2002; Karanian et al. 2005a). Transverse slices $(400 \mu \mathrm{m})$ were quickly prepared, and the hippocampal slices were kept in ice-cold buffer until groups of eight to nine slices were placed on the Biopore PTFE membrane of each Millicell-CM culture insert (Millipore, Billerica, MA). Media was changed periodically, consisting of $50 \%$ basal medium Eagle (Sigma-Aldrich, St. Louis, MO), 25\% Earle's balanced salts (Sigma-Aldrich), 25\% horse serum (Gemini BioProducts, Sacramento, CA), and defined supplements, as described previously (Bendiske et al. 2002; Butler et al. 2007). The hippocampal slices were maintained at $37{ }^{\circ} \mathrm{C}$ in $5 \% \mathrm{CO}_{2}$ enriched atmosphere for an 18-20-day maturation period before being treated with different agents.

Cultured hippocampal slices were pre-treated for $1 \mathrm{~h}$ with media plus $0.02 \%$ DMSO, in the absence or presence of $20 \mu \mathrm{M}$ AM5206. Slices were subsequently treated with vehicle or $200 \mu \mathrm{M}$ Pxn (Tocris, Ellisville, MO), freshly prepared, for $24 \mathrm{~h}$ in the absence or presence of $20 \mu \mathrm{M}$ of AM5206. Note, AM5206 is 280 - to 480 -fold more selective for FAAH than monoacylglycerol lipase (Naidoo et al. 2011). After the treatments, cultured slices were fixed in $4 \%$ paraformaldehyde for histology or gently harvested from the inserts using icecold isosmotic buffer containing $0.32 \mathrm{M}$ sucrose, $5 \mathrm{mM}$ HEPES (pH 7.4), 1 mM EDTA, and 1 mM EGTA.

A group of cultured hippocampal slices fixed in 4\% paraformaldehyde were rinsed in PBS, blocked with 5\% BSA in PBS containing $0.1 \%$ TX-100 for $1 \mathrm{~h}$. Following, the tissue was incubated with anti-NeuN antibody (1:200, Abcam, Cambridge, MA) and by the secondary antibody step (1:750, Alexa Fluor-488, Molecular Probe, Thermo Scientific, Rockford, IL), and Neurotrace 640/660 deep-red fluorescent Nissl stain (1:100, Thermo-Scientific). A Nikon Eclipse T $i$ confocal Microscope (Nikon Instruments Inc., Melville, NY) was used for imaging. Analysis and edition were performed with NIS-Elements AR (Nikon).

Sprague-Dawley male rats were injected intraperitoneal (i.p.) with vehicle or with AM5206 in a DMSO-PBS solution $(50 / 50 \%)$, for a dose of $8.0 \mathrm{mg} / \mathrm{kg}$. Following, the rats were immediately injected subcutaneous (s.c.) with either vehicle (100\% corn oil, Sigma-Aldrich) or a dose of $0.3 \mathrm{mg} / \mathrm{kg}$ Pxn (Tocris) for assessment of the acute response. The animals were returned to their cages, and seizure activities were recorded for $4 \mathrm{~h}$. Then, at $24 \mathrm{~h}$ after last injections, the rats were subjected to behavioral assessment. The seizure activity was rated by observers blinded to the treatment groups at 15 -min intervals for a maximum of $4 \mathrm{~h}$ according to a well-established rating scale (Karanian et al. 2007; Naidoo et al. 2012). Note, Pxn dosage range was initially tested between 0.3 and $0.8 \mathrm{mg} /$ $\mathrm{kg}$. The dose of $0.3 \mathrm{mg} / \mathrm{kg}$ was chosen to elicit a brain insult with no issues of lethality. A dose of $0.8 \mathrm{mg} / \mathrm{kg}$ produced unsurvived seizures, prolonged higher than $20 \mathrm{~h}$.

For behavioral testing, balance and coordination were examined using behavioral paradigms $24 \mathrm{~h}$ after injection procedures as previously described (Karanian et al. 2005b, 2007). In the rota-rod test, rats were first trained on a rotating cylinder 
(15 rpm) for 10 consecutive trials with a maximum of $10 \mathrm{~s}$ per trial. The animals were allowed to rest, and then re-tested on the rota-rod for three consecutive trials up to a maximum of $2 \mathrm{~min}$. The time the animal stayed on the rod was recorded and averaged across the three trials. The second behavioral paradigm was the balance beam in which rats were placed in the middle of a suspended wooden bar (1.2-cm diameter) positioned $15 \mathrm{~cm}$ above a padded surface. During this task, animals were assessed for the time before falling from the bar, up to a maximum of $30 \mathrm{~s}$ per trial for each of three consecutive trials. Following, the animals were anesthetized with isoflurane (Abbott Laboratories, Chicago, IL), and the brains were quickly removed and placed in ice-cold buffer with protease inhibitors. The brain regions, hippocampus, and frontal cortex were dissected out and kept frozen until to be assessed by immunoblot.

Proteins were immunoblotted using the tissue from hippocampal slice cultures and young rats. The samples were homogenized in lysis buffer consisting of $15 \mathrm{mM}$ HEPES (pH 7.4), $0.5 \mathrm{mM}$ EDTA, $0.5 \mathrm{mM}$ EGTA with protease inhibitors cocktail (Sigma-Aldrich), sonicated, and followed by protein concentration determination using Pierce BCA Protein Assay (ThermoScientific). Equal amounts of protein sample were denatured for $5 \mathrm{~min}$ at $100{ }^{\circ} \mathrm{C}$, separated by SDS-PAGE, and transferred to nitrocellulose membranes (Bio-Rad, Hercules, CA). Next, membranes were blocked with 5\% non-fat dry milk for $1 \mathrm{~h}$ at room temperature, followed by incubation with primary antibodies against GluR1 (1:1000), synapsin II (1:1000), and NCAM (1:200) from Millipore; actin (1:500, SigmaAldrich); $\beta 1$ integrin (1:1000), synaptophysin (1:1000) and 4HNE (1:500) from Abcam (Cambridge, MA). For secondary antibody steps, goat anti-rabbit $\operatorname{IgG}(1: 1800)$ and goat antimouse $\operatorname{IgG}(1: 1600)$ conjugated with alkaline phosphatase were from Bio-Rad, or horseradish peroxidase-conjugated goat antirabbit $\operatorname{IgG}(1: 10,000)$ and goat anti-mouse $\operatorname{IgG}(1: 6000)$ were from Amersham GE Healthcare (Pittsburgh, PA); these steps were used in $2 \%$ milk and incubated for $1 \mathrm{~h}$ at room temperature. Development of antigens used either the 5-bromo-4chloro-3-indolyl phosphate and nitroblue tetrazolium substrate system or enhanced chemiluminescence reagent, followed by analysis with Amersham Imager 600 (GE Healthcare). Stained antigens were scanned at high resolution to determine integrated optical density (IOD) with BIOQUANT software (R\&M Biometrics, Nashville, TN). All the results were evaluated by unpaired $t$ tests or analyses of variance (ANOVA) followed by post hoc tests using Prism software (GaphPad, San Diego, CA).

\section{Results}

Hippocampal slice cultures are well known for their maintenance of three-dimensional native organization and circuitry. The distinct laminar subfield and neuronal morphology of the hippocampus were assessed in the slice (see Fig. 1). After the
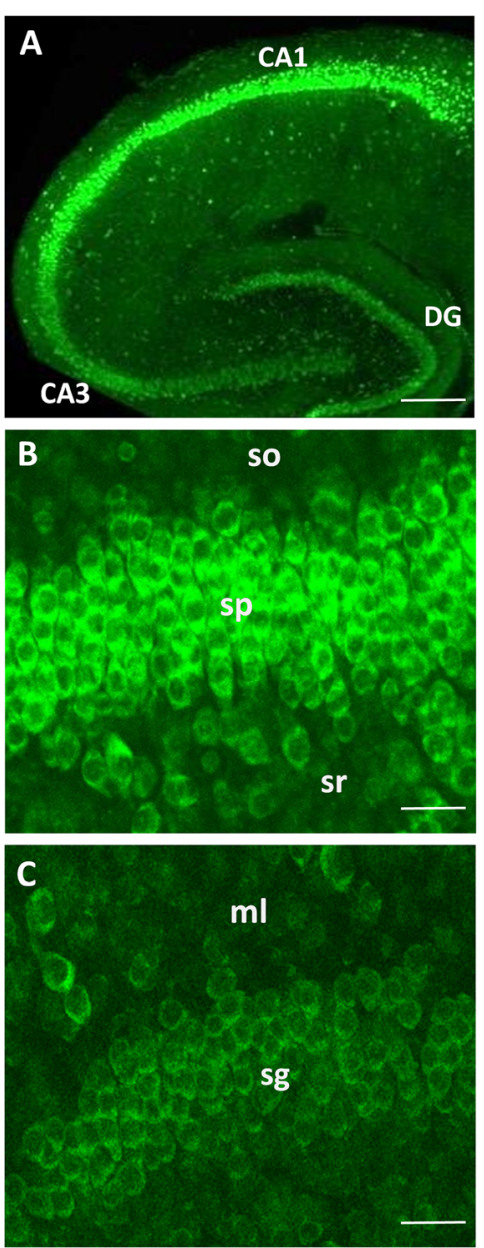

Fig. 1 Hippocampal slice cultures maintain native neuronal organization and density. Transverse slices of hippocampi were prepared from brain tissue of 11-day-old rat pups and placed on Biopore inserts. The tissue was immunostained with anti-NeuN antibody, and confocal imaging showed the appearance of the disparate hippocampus subfields (a). High magnification view of fluorescent Nissl stain shows normal neuronal morphology after 18 days in culture in the stratum pyramidal layer $(\mathrm{sp} ; \mathbf{b})$ and stratum granulosum layer (sg; c), pseudocolored in green. Size bar: a $500 \mu \mathrm{m}$ and $\mathbf{b}$ and $\mathbf{c} 25 \mu \mathrm{m}$

maturation period of 18 days, mature brain tissue exhibiting native features at high magnification was visualized in the stratum pyramidale (Fig. 1b) and the stratum granulosum layer of the dentate gyrus (Fig. 1c). To study direct effects of an anticholinesterase on this brain tissue, the mature hippocampal slices were exposed to Pxn dosages of 10, 50, 100, and $200 \mu \mathrm{M}$. Only the $200 \mu \mathrm{M}$ dose exhibited a consistent effect in regards the synaptic decline at $24 \mathrm{~h}$ post-application, leading to a reduction of the presynaptic marker synapsin IIb (syn IIb) (Fig. 2a). Note, syn IIb was reduced by $64 \%$ in Pxntreated slice cultures (Fig. 2b, $p=0.0006$ ). The synaptophysin levels were also decreased by the acute Pxn exposure (see Fig. 3a). Interestingly, the slices exhibiting Pxn-induced presynaptic decline did not show any reduction in the postsynaptic marker GluR1 (Fig. 2a and c). Another sign of Pxn-induced 
A
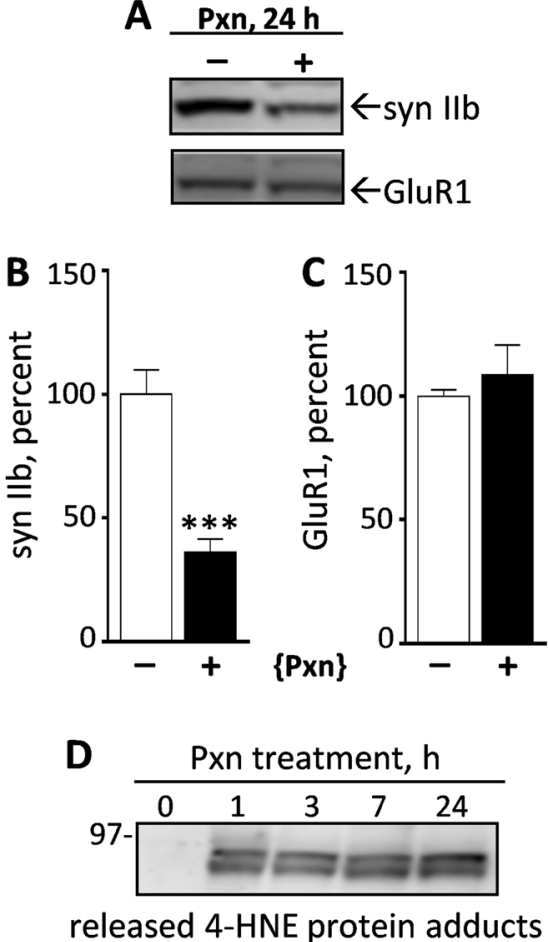

Fig. 2 Pxn acute insult specifically reduces presynaptic marker and displays oxidative stress products in hippocampal slice cultures. Slices of hippocampus were maintained in culture for 18 days; then, the tissue was exposed to $200 \mu \mathrm{M}$ Pxn for $24 \mathrm{~h}$ alongside vehicle-treated slices. The slices were harvested into three groups of six to eight each, and equal protein aliquots assessed for synaptic markers synapsin IIb (syn IIb) and GluR1 by immunoblot (a). Immunoreactivities were normalized to their respective controls, and percent \pm SEM are shown for syn IIb (b) and GluR1 (c). Unpaired $t$ test: $* * * p<0.001$. Media samples from Pxntreated hippocampal slice cultures were assessed for 4-HNE-protein adducts, an indicator of oxidative stress (d). Position of molecular weight standard is shown $(\mathrm{kDa})$

cytotoxicity was the oxidative stress-related production of stable 4-HNE-protein adducts released into the media (Fig. 2d). Together, these results suggest that $200 \mu \mathrm{M}$ Pxn may cause neuronal compromise and increase oxidative stress products in cultured hippocampal slices, and presynaptic markers appear to be more susceptible than postsynaptic markers.

As a test for neuroprotection, the hippocampal slices exposed to Pxn were also treated with the FAAH inhibitor AM5206, a compound that promotes protective endocannabinoid signaling and reduces excitotoxic damage (see Karanian et al. 2005b; Naidoo et al. 2011). Here, we used the same concentration of AM5206 used previously to preferentially diminish FAAH activity as compared to little effect on MAGL (Naidoo et al. 2011). First, cultured hippocampal slices were pre-treated with AM5206 or vehicle for $1 \mathrm{~h}$; then, they were subjected to Pxn exposure with the continued presence of AM5206 or vehicle for $24 \mathrm{~h}$. The data show that AM5206 attenuates Pxn-induced synaptic deterioration by restoring syn IIb levels, and similar protection was found for synaptophysin (Fig. 3a). The syn IIb levels were enhanced
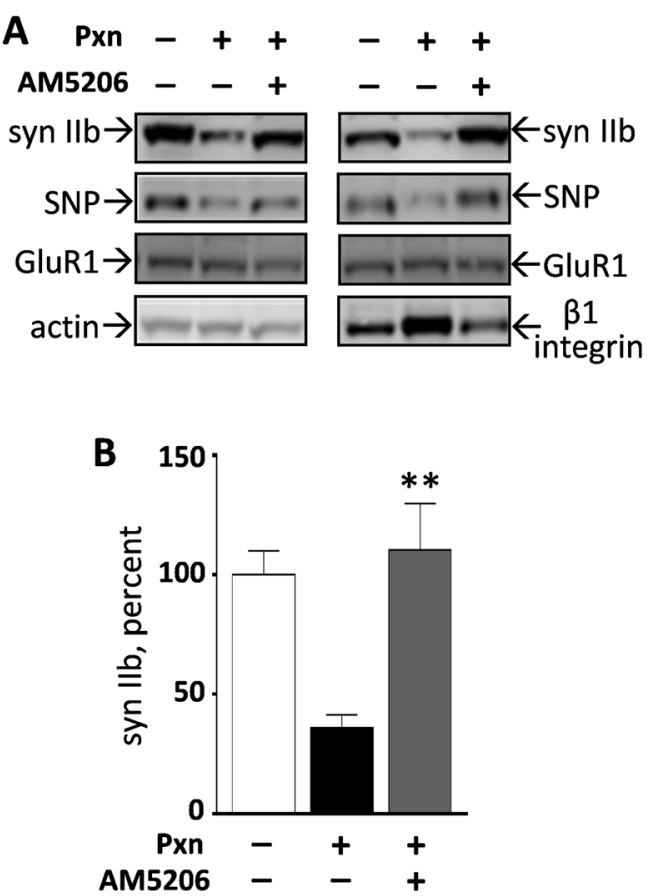

Fig. 3 Endocannabinoid enhancement pathway by FAAH inhibitor prevents Pxn-induced presynaptic deterioration. Cultured hippocampal slices were pre-treated with $20 \mu \mathrm{M}$ of AM5206, a fatty acid amide hydrolase (FAAH) inhibitor, for $1 \mathrm{~h}$ alongside vehicle-treated slices followed by $200 \mu \mathrm{M}$ of Pxn with AM5206 or vehicle for $24 \mathrm{~h}$. The slices were harvested into three groups of six to eight slices each and per condition, and equal protein aliquots assessed for synapsin IIb (syn IIb), synaptophysin (SNP), GluR1, $\beta 1$ integrin, and the load control actin by immunoblot (a). The syn IIb levels were normalized to their respective controls, and percent \pm SEM are shown (b). Unpaired $t$ test: $* * p<0.01$ compared to Pxn-treated slices

3-fold when AM5206 was present during Pxn exposure in comparison to Pxn alone (Fig. 3b, $p<0.01$ ).

As expected from the above results, no change in GluR1 was found in the Pxn-treated slices, and the addition of AM5206 also had no negative effect on the postsynaptic marker (Fig. 3a). Interestingly, Pxn-induced presynaptic marker loss was consistently found associated with aberrant $\beta 1$ integrin activation. As part of its neuroprotective action, AM5206 modulated $\beta 1$ integrin responses to levels comparable to those found in control slices (Fig. 3a, $p=0.023$ ). These data together attribute the AM5206 protective response, which specifically targets FAAH to modulate endogenous cannabinoid signaling (Naidoo et al. 2011). Noteworthy, evidence of protective responses by the endocannabinoid pathway was also shown in other studies of excitotoxicity (Karanian et al. 2005b; Sagar et al. 2010; Naidoo et al. 2011, 2012; Shubina et al. 2017).

Next, we tested whether the protective effects of AM5206 in the slice model translate to protection in an animal model. A dose-dependent effect for reducing seizure severity was first established for AM5206 in a 
kainate rat model in which the kainate-treated animals expressed seizure scores of $2.8 \pm 0.24(n=12)$, whereas rats that also received $1(n=4), 5(n=5)$, or $8 \mathrm{mg} / \mathrm{kg}$ AM5206 $(n=8)$ exhibited 25,43 , and $79 \%$ reductions in mean seizure scores, respectively (ANOVA: $p<0.0001$ ). In addition, a dosage range of $0-15 \mathrm{mg} / \mathrm{kg}$ for safety assessment was previously tested, and no behavioral abnormalities or seizures were observed across the 14-day period, including any alteration in alanine aminotransferase activity and blood urea nitrogen levels (Naidoo et al. 2011). Accordingly, the $8 \mathrm{mg} / \mathrm{kg}$ effective dose was tested against Pxn exposure. Here, Pxn-treated rats exhibited prolonged tonic-clonic convulsions and seizures that were assessed with a 0-6 rating scale (Fig. 4a). Interestingly, seizure severity was reduced by $86 \%$ when the FAAH inhibitor AM5206 was pre-administered to the Pxntreated animals at the protective $8 \mathrm{mg} / \mathrm{kg}$ dose (Fig. 4a, $p<0.001$ ). Consistent with seizure attenuation, AM5206 also displayed behavioral protection; while Pxn exposure caused a reduction in performance on the rota-rod paradigm to assess balance and coordination, Pxn rats that received pre-treatment with the AM5206 FAAH inhibitor displayed nearly complete recovery in rota-rod performance (Fig. $4 \mathrm{~b}, p<0.01$ ). In addition, Pxn reduced balance beam performance, and significant improvement was shown for those animals pre-treated with AM5206 (Fig. 4c). Therefore, AM5206 attenuated seizures and significantly improved performance in both behavioral paradigms.

To further evaluate the neuroprotection in vivo, we tested whether AM5206 provides synaptic protection against the Pxn administration. The evidence of disruption of synaptic integrity found in slice cultures after Pxn exposure was also found in Pxn-treated rats. Pxn reduced syn IIb levels in two vulnerable brain regions - the hippocampus and frontal cortex. In contrast, AM5206 protected the deterioration of the presynaptic marker in both brain regions (Fig. 5a). Also, as previously reported, the reduction in kainate-induced seizures by $8 \mathrm{mg} / \mathrm{kg}$ AM5206 was associated with synaptic and behavioral protection (Naidoo et al. 2011). FAAH inhibitor also exhibited an ability to protect against secondary events associated with the Pxn-induced cytotoxicity, in particular the aberrant activation of $\beta 1$ integrin. As found in slice model, cortical and hippocampal samples from Pxn-treated rats exhibited up to 3 -fold increases in $\beta 1$ integrin levels, and these levels were significantly reduced by AM5206 (Fig. $5 \mathrm{a}, p=0.02$ ). As a final note, NCAM, a postsynaptic marker indicator, was unchanged in both brain regions - the hippocampus and neocortex (Fig. 5b). Such evidence further suggests that the synaptic vulnerability caused by Pxn involves presynaptic markers as previously observed in vitro.

Finally, to address whether AM5206 is effective in reducing oxidative damage both in vitro and in vivo,
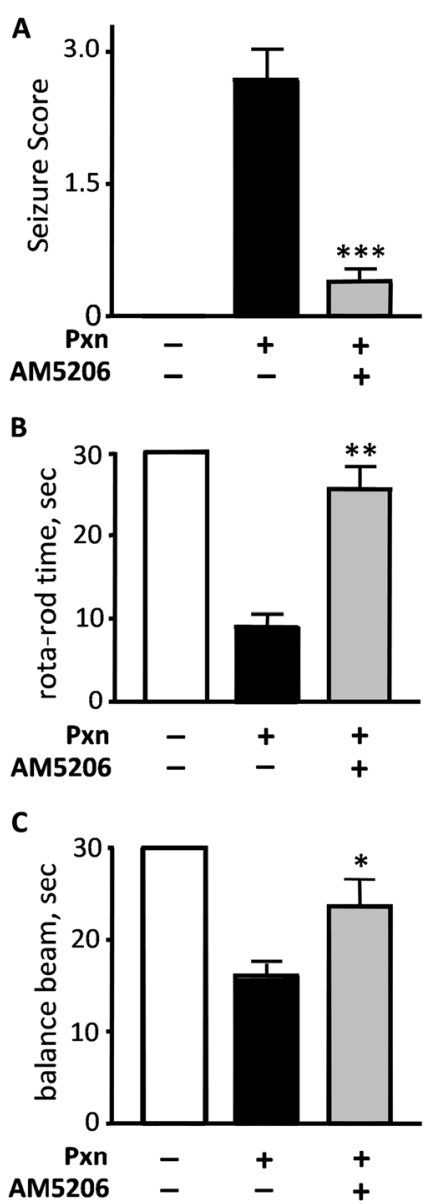

Fig. 4 In vivo Pxn exposure triggers seizures and behavioral compromise although attenuated by AM5206 treatment. Young Sprague-Dawley rats were injected i.p. with AM5206 $(n=5)$ alongside vehicle-treated rats, followed immediately injected with Pxn s.c. $(n=4)$. Seizures were scored by blinded raters over a 1 -h period \pm SEM (a). The control group was normalized across 10 animals. Unpaired $t$ test: $* * * p<0.001$ compared to Pxn group. Next, at $24 \mathrm{~h}$ post-injection, the animals were evaluated with a rota-rod paradigm to assess their coordination, as determined by the mean time \pm SEM maintaining coordinated movement on a rod rotating at $15 \mathrm{rpm}(\mathbf{b})$. Also, the animals were placed on a narrow, suspended bar to assess the balance beam test, determined by the mean time $\pm \operatorname{SEM}(\mathbf{c})$. Unpaired $t$ test: $* p<0.05, * * p<0.01$ compared to Pxn group

we assessed 4-HNE-protein adducts of $76 \mathrm{kDa}$. The 4HNE adducts were effectively reduced due to AM5206 treatment as compared to Pxn alone in the slice model (Fig. 6a). Similarly, hippocampi from Pxn-treated rats exhibited increased amounts of 4-HNE-protein adducts, and the presence of AM5206 displayed protection against the harmful production of 4-HNE modifications in the Pxn-treated rats (Fig. 6b and c).

\section{Discussion}

The present study indicates that the neurotoxin Pxn elicits a similar profile of synaptic toxicity in vitro and in vivo with 


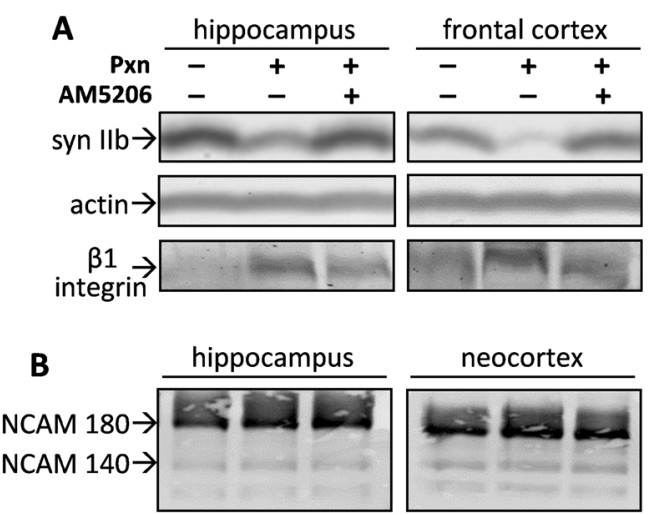

Fig. 5 FAAH inhibitor prevents synaptic vulnerability after Pxn-induced synaptotoxicity in vivo. Young Sprague-Dawley rats were injected i.p. with AM5206 $(n=5)$ alongside vehicle-treated rats, followed immediately injected with Pxn s.c. $(n=4)$. Brain regions were dissected and assessed by the immunoblots for synapsin IIb (syn IIb), load control actin, and $\beta 1$ integrin (a). Similarly, immunoblots were also assessed for neural cell adhesion molecule (NCAM, b)

regards to the selective reduction in the presynaptic marker syn $\mathrm{IIb}$. Moreover, the in vivo decline in syn IIb levels was observed in those Pxn-treated animals that presented seizures and behavioral deficits. With the direct application of Pxn to the brain
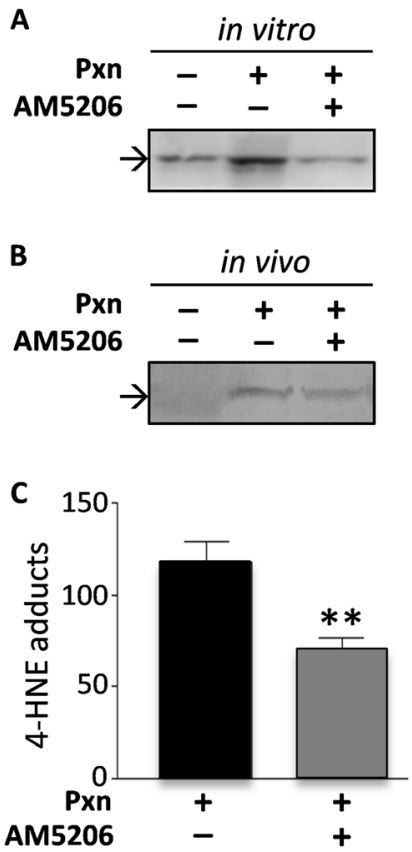

Fig. 6 FAAH inhibitor elicits protection against oxidative stress products after Pxn insult. Products of lipid peroxidation were assessed by 4-HNEprotein adducts of $76 \mathrm{kDa}$ (see arrows). First, cultured hippocampal slices were pre-treated with AM5206 for $1 \mathrm{~h}$ alongside vehicle-treated slices followed by Pxn with AM5206 or vehicle for $24 \mathrm{~h}$, then harvested into groups of six to eight slices each and assessed by immunoblot for 4-HNE (a). In parallel, young Sprague-Dawley rats were injected i.p. with AM5206 alongside vehicle-treated rats, followed immediately injected with Pxn s.c. in both groups of three each. Following, the hippocampi were dissected and assessed by immunoblots for 4-HNE-protein adducts (b), and the levels for the in vivo study were plotted as mean percent of control \pm SEM (c). Unpaired $t$ test: $* * p=0.01$ compared to Pxn group tissue using slice cultures of hippocampi, the toxin produced a significant reduction in syn IIb levels by $64 \%$. This synaptic disruption induced by Pxn can be compared to synaptic marker declines reported in various excitotoxicity studies using hippocampal slice culture (Bahr et al. 2002; Karanian et al. 2005b; Naidoo et al. 2012; Quintana et al. 2015; Piwońska et al. 2016). It is noteworthy that synaptic damage has been postulated as an early sign of toxicity that leads to neuronal dysfunction and memory impairment (Raveh et al. 2002, 2003; Munirathinam and Bahr 2004), and the hippocampus is a vulnerable brain region susceptible to organophosphate compounds (Crino et al. 2002; Harrison et al. 2004), particularly due to the highdensity cholinergic and glutamatergic innervations.

Pxn exposure causes the accumulation of acetylcholine and subsequent signs of hypercholinergic syndrome (Marrs 1993; Jamal 1997; Sánchez-Santed et al. 2004). Consistent with the present results, disturbances associated with Pxn-mediated excitotoxicity occurs principally through presynaptic mechanisms (Kozhemyakin et al. 2010). Here, the hippocampal slice model expressed a selective reduction in the syn IIb isoform after Pxn exposure. Synapsin II is a synaptic vesicleassociated protein suggested to be a central element in the organization and regulation of the active pool of vesicles in nerve terminals (Chi et al. 2001). Many of the molecular features of synapsin II suggest that it plays a regulatory role in vesicular mobilization involved in short-term synaptic depression and facilitation. In contrast to the syn IIb effect, no change was found regarding the postsynaptic marker GluR1 in Pxn-treated slices. However, it has been shown that the potent neurotoxin soman, another organophosphate, elicited a reduction in GluR1 in hippocampal slices (Munirathinam and Bahr 2004). In agreement with the Pxn effects on GluR1, Mohammadi et al. (2016) observed no change in the mRNA expression and protein levels of the neuronal glutamate transporter in paraoxon-treated animals.

Assessment of the FAAH inhibitor AM5206 found substantial protection against Pxn exposure by preserving syn Ilb levels in hippocampal slice cultures. Note, compromised syn Ilb levels were increased 3-fold by AM5206, repairing the presynaptic marker to levels comparable to those in control slices. Similar protection was evident with cannabinoid signaling activated by low-dose $\Delta$ 9-tetrahydrocannabinol (THC), reversing the agerelated decline in cognitive performance accompanied by enhanced expression of synapsin and synaptophysin in vivo (Bilkei-Gorzo et al. 2017). In addition, the 4-HNE-protein adducts generated after Pxn exposure were decreased by the FAAH inhibitor. The 4-HNE modification is widely accepted as a marker of oxidative stress (Chen and Niki 2006), and 4HNE bound to proteins is considered the most likely form of 4HNE in cells. Thus, the AM5206 FAAH inhibitor appears to offer an approach for modulating endocannabinoid signaling to promote protection against Pxn-induced presynaptic vulnerability and oxidative stress. The results further indicate that FAAH 
inhibition influences endogenous cannabinoids for homeostatic regulation. Blocking $\mathrm{FAAH}$ is known to reduce endocannabinoid deactivation, and the enhanced endocannabinoid system mediates protection through pathways linked to the CB1 receptor (see Galve-Roperh et al. 2002; Molina-Holgado et al. 2005; Karanian et al. 2005b, 2007).

Hippocampal slice cultures are valuable for the analysis of neuropathological events by reproducing the toxicological changes as found in vivo (see Thompson et al. 1996; Karanian et al. 2005a; Naidoo et al. 2012). The molecular indicators of toxicity in the slice model were observed in the Pxn-treated rat model as well. As in the slice culture results, the neuroprotection assessed for FAAH inhibition also translated to the in vivo Pxn model, thus indicating the usefulness of screening studies in cultured tissue slices. Acute exposure to anticholinesterase agents is well known to evoke seizure activity leading to neuronal damage (Kozhemyakin et al. 2010, Ruban et al. 2015; Shrot et al. 2015). As expected, in vivo Pxn exposure in the current study led to seizure events and associated disruption in behavior. The AM5206 FAAH inhibitor decreased the seizure scores in Pxntreated rats, producing suppression of seizure severity and attenuation of behavioral impairment in correspondence with evidence of synaptic protection.

In both the slice and rat models, protecting presynaptic marker levels was tied to the prevention and/or recovery of Pxn-induced alterations to the $\beta 1$-class integrin. The latter is part of a large family of extracellular matrix receptors involved in a wide range of synaptic integrin functions (Park and Goda 2016). The $\beta 1$ integrin responses may be responsible for regulating the readily releasable pool of synaptic vesicles (Huang et al. 2006), thus connecting to the presynaptic vulnerability described. Noting that neuronal stimulation activates $\beta 1$ integrins in hippocampal synapses (Babayan et al. 2012), the excitatory over-activation through Pxn toxicity may be initiating a level of synaptic cofilin and other signaling above that which maintains physiological neurotransmission and plasticity (see Woo et al. 2015; Lauterborn et al. 2017). Of the two cannabinoid CB1 receptor (CB1R) signaling cascades recently identified (Wang et al. 2017), perhaps the protective endocannabinoid action of AM5206 occurs through the cascade that is independent of cooperative $\mathrm{CBR} 1 / \beta 1$ integrin responses.

Pxn exposure produced seizure events and behavioral compromise in correspondence with synaptic disturbance. AM5206 protected against the Pxn-induced deficits in balance and coordination, and the improved behavior was accompanied by synaptic protection indicated by levels of syn IIb. These data suggest that the FAAH inhibitor is able to prevent presynaptic deterioration, thereby reducing functional deficits. FAAH has been localized postsynaptically within many brain regions, including hippocampal and neocortical neuronal cell bodies (see Egertova et al. 2003). In Pxn-treated rats, the presynaptic marker syn IIb was reduced whereas 4-HNE-protein adducts were enhanced, presenting the same profile as found in the hippocampal slice model. FAAH inhibition appears to reduce the sign of oxidative stress and prevent the negative impact on synaptic function. Note, oxidative damage along with presynaptic vulnerability during seizure events may be a pathogenic combination that disrupts cellular homeostasis. Recently, an in vivo study showed that the beneficial effects of THC cannabinoid signaling are dependent on glutamatergic CB1 receptors (Bilkei-Gorzo et al. 2017); thus, it is interesting that Pxn did not reduce the glutamatergic marker GluR1, perhaps signifying that this type of toxin has a higher probability of being treated through the cannabinergic pathway and that FAAH inhibitors can be helpful to combat neuropathogenic cascades.

Acknowledgements This material is based upon work supported by the US Army Research Office and the Department of Defense Research and Education Program under grant number W911NF-15-1-0432 (BAB). The research was also supported by National Institutes of Health (NIH) grants DA009158 (AM), DA003801 (AM), and DA007215 (AM) and in part by National Institutes of Health-Research Initiative for Scientific Enhancement (NIH-RISE) grant 5R25GM077634-04 (UNCP). The funding agencies had no role in study design, data collection and analysis, or decision to publish. We thank Jeannie Hwang, Christopher Long, Kathlyn Stephens, and Wynne Kelly for excellent assistance in the laboratory.

\section{References}

Babayan AH, Kramár EA, Barrett RM, Jafari M, Häettig J, Chen LY, Rex CS, Lauterborn JC, Wood MA, Gall CM, Lynch G (2012) Integrin dynamics produce a delayed stage of long-term potentiation and memory consolidation. J Neurosci 32:12854-12861

Bahr BA, Bendiske J, Brown QB, Munirathinam S, Caba E, Rudin M, Urwyler S, Sauter A, Rogers G (2002) Survival signaling and selective neuroprotection through glutamatergic transmission. Exp Neurol 174:137-147

Bahr BA, Karanian DA, Makanji SS, Makriyannis A (2006) Targeting the endocannabinoid system in treating brain disorders. Expert Opin Investig Drugs 15:351-365

Bendiske J, Caba E, Brown QB, Bahr BA (2002) Intracellular deposition, microtubule destabilization, and transport failure: an "early" pathogenic cascade leading to synaptic decline. J Neuropathol Exp Neurol 61:640-650

Bilkei-Gorzo A, Albayram O, Draffehn A, Michel K, Piyanova A, Oppenheimer H, Dvir-Ginzberg M, Rácz I, Ulas T, Imbeault S, Bab I, Schultze JL, Zimmer A (2017) A chronic low dose of $\Delta 9$ tetrahydrocannabinol (THC) restores cognitive function in old mice. Nat Med 23:782-787

Butler D, Bendiske J, Michaelis ML, Karanian DA, Bahr BA (2007) Microtubule-stabilizing agent prevents protein accumulationinduced loss of synaptic markers. Eur J Pharmacol 562:20-27

Chen ZH, Niki E (2006) 4-hydroxynonenal (4-HNE) has been widely accepted as an inducer of oxidative stress. Is this the whole truth about it or can 4-HNE also exert protective effects? Int Union Biochem Mol Biol 58:372-373

Chi P, Greengard P, Ryan TA (2001) Synapsin dispersion and reclustering during synaptic activity. Nat Neurosci 4:1187-1193

Crino PB, Jin H, Shumate MD, Robinson MB, Coulter DA, BrooksKayal AR (2002) Increased expression of the neuronal glutamate transporter (EAAT3/EAAC1) in hippocampal and neocortical epilepsy. Epilepsia 43:211-218 
Egertova M, Cravatt BF, Elphick MR (2003) Comparative analysis of fatty acid amide hydrolase and CB1 cannabinoid receptor expression in the mouse brain: evidence of a widespread role for fatty acid amide hydrolase in regulation of endocannabinoid signaling. Neuroscience 119:481-496

Fernandez-Ruiz J, Garcia C, Sagredo O, Gomez-Ruiz M, de Lago E (2010) The endocannabinoid system as a target for the treatment of neuronal damage. Expert Opin Ther Targets 14:387-404

Galve-Roperh I, Rueda D, Gomez del Pulgar T, Velasco G, Guzman M (2002) Mechanism of extracellular signal-regulated kinase activation by the CB(1) cannabinoid receptor. Mol Pharmacol 62:1385-1392

Harrison PK, Sheridan RD, Green AC, Scott IR, Tattersall JE (2004) A guinea pig hippocampal slice model of organophosphate-induced seizure activity. J Pharmacol Exp Ther 310:678-686

Huang Z, Shimazu K, Woo NH, Zang K, Müller U, Lu B, Reichardt LF (2006) Distinct roles of the $\beta 1$-class integrins at the developing and the mature hippocampal excitatory synapse. J Neurosci 26:11208-11219

Hwang J, Adamson C, Butler D, Janero DR, Makriyannis A, Bahr BA (2010) Enhancement of endocannabinoid signaling by fatty acid amide hydrolase inhibition: a neuroprotective therapeutic modality. Life Sci 86:615-623

Jamal GA (1997) Neurological syndromes of organophosphorus compounds. Adverse Drug React Toxicol Rev 16:133-170

Karanian DA, Brown QB, Makriyannis A, Bahr BA (2005a) Blocking cannabinoid activation of FAK and ERK1/2 compromises synaptic integrity in hippocampus. Eur J Pharmacol 508:47-56

Karanian DA, Brown QB, Makriyannis A, Kosten TA, Bahr BA (2005b) Dual modulation of endocannabinoid transport and fatty acid amide hydrolase protects against excitotoxicity. J Neurosci 25:7813-7820

Karanian DA, Karim SL, Wood JT, Williams JS, Lin S, Makriyannis A, Bahr BA (2007) Endocannabinoid enhancement protects against kainic acid-induced seizures and associated brain damage. $\mathrm{J}$ Pharmacol Exp Ther 322:1059-1066

Kathuria S, Gaetani S, Fegley D, Valiño F, Duranti A, Tontini A, Mor M, Tarzia G, La Rana G, Calignano A, Giustino A, Tattoli M, Palmery M, Cuomo V, Piomelli D (2003) Modulation of anxiety through blockade of anandamide hydrolysis. Nat Med 9:76-81

Kozhemyakin M, Rajasekaran K, Kapur J (2010) Central cholinesterase inhibition enhances glutamatergic synaptic transmission. J Neurophysiol 103:1748-1757

Lauterborn JC, Kramár EA, Rice JD, Babayan AH, Cox CD, Karsten CA, Gall CM, Lynch G (2017) Cofilin activation is temporally associated with the cessation of growth in the developing hippocampus. Cereb Cortex 27:2640-2651

Marrs TC (1993) Organophosphate poisoning. Pharmacol Ther 58:51-66

Mohammadi M, Ghani E, Ghasemi A, Khoshbaten A, Asgari A (2008) Synaptosomal GABA uptake decreases in paraoxon-treated rat brain. Toxicology 244:42-48

Mohammadi M, Zare Z, Allah-Moradi E, Vaezi N, Valadan R, Tehrani M (2016) Alterations in mRNA and protein expression of glutamate transporters in rat hippocampus after paraoxon exposure. Neurotoxicology 57:251-257

Molina-Holgado F, Pinteaux E, Heenan L, Moore JD, Rothwell NJ, Gibson RM (2005) Neuroprotective effects of the synthetic cannabinoid HU210 in primary cortical neurons are mediated by phosphatidylinositol 3-kinase/AKT signaling. Mol Cell Neurosci 28:189-194

Munirathinam S, Bahr BA (2004) Repeated contact with subtoxic soman leads to synaptic vulnerability in hippocampus. J Neurosci Res 77: 739-746

Naidoo V, Nikas SP, Karanian DA, Hwang J, Zhao J, Wood JT, Alapafuja SO, Vadivel SK, Butler D, Makriyannis A, Bahr BA (2011) A new generation fatty acid amide hydrolase inhibitor protects against kainite-induced excitotoxicity. J Mol Neurosci 43:493-502

Naidoo V, Karanian DA, Vadivel SK, Locklear JR, Wood JT, Nasr M, Quizon PMP, Graves EE, Shukla V, Makriyannis A, Bahr BA
(2012) Equipotent inhibition of fatty acid amide hydrolase and monoacylglycerol lipase-dual targets of the Endocannabinoid system to protect against seizure pathology. Neurotherapeutics 9:810-813

Park YK, Goda Y (2016) Integrins in synapse regulation. Nat Rev Neurosci 17:745-756

Piwońska M, Szewczyk A, Schröder UH, Reymann KG, Bednarczyk I (2016) Effectors of large-conductance calcium-activated potassium channel modulate glutamate excitotoxicity in organotypic hippocampal slice cultures. Acta Neurobiol Exp 76:20-31

Prager EM, Figueiredo TH, Long RP 2nd, Aroniadou-Anderjaska V, Apland JP, Braga MF (2015) LY293558 prevents soman-induced pathophysiological alterations in the basolateral amygdala and the development of anxiety. Neuropharmacology 89:11-18

Quintana P, Soto D, Poirot O, Zonouzi M, Kellenberger S, Muller D, Chrast R, Cull-Candy SG (2015) Acid-sensing ion channel 1a drives AMPA receptor plasticity following ischaemia and acidosis in hippocampal CA1 neurons. J Physiol 593:4373-4386

Raveh L, Weissman BA, Cohen G, Alkalay D, Rabinovitz I, Sonego H, Brandeis R (2002) Caramiphen and scopolamine prevent somaninduced brain damage and cognitive dysfunction. Neurotoxicology 23:7-17

Raveh L, Brandeis R, Gilat E, Cohen G, Alkalay D, Rabinovitz I, Sonego H, Weissman BA (2003) Anticholinergic and antiglutamatergic agents protect against soman-induced brain damage and cognitive dysfunction. Toxicol Sci 75:108-116

Reynolds D (2017) EPA reversal of pesticide ban signals shift away from using human data. Inside EPA's Risk Policy Report 24:14. https://0search-proquest-com.bravecat.uncp.edu/docview/1883574316? accountid=13153. Accessed 4 Apr 2017

Rosenberg EC, Patra PH, Whalley BJ (2017) Therapeutic effects of cannabinoids in animal models of seizures, epilepsy, epileptogenesis, and epilepsy-related neuroprotection. Epilepsy Behav 70:319-327

Rotenberg JS, Newmark J (2003) Nerve agent attacks on children: diagnosis and management. Pediatrics 112:648-658

Ruban A, Biton IE, Markovich A, Mirelman D (2015) MRS of brain metabolite levels demonstrates the ability of scavenging of excess brain glutamate to protect against nerve agent induced seizures. Int J Mol Sci 16:3226-3236

Sagar DR, Jhaveri MD, Richardson D, Gray RA, de Lago E, FernandezRuiz J, Barret DA, Kendhall DA, Chapman V (2010) Endocannabinoid regulation of spinal nociceptive processing in a model of neuropathic pain. Eur J Neurosci 31:1414-1422

Sánchez-Santed F, Cañadas F, Flores P, López-Grancha M, Cadona D (2004) Long-term functional neurotoxicity of paraoxon and chlorpyrifos: behavioral and pharmacological evidence. Neurotoxicol Teratol 26:305-317

Shrot S, Tauber M, Shiyovich A, Milk N, Rosman Y, Eisenkraft A, Kadar T, Kassirer M, Cohen Y (2015) Early brain magnetic resonance imaging can predict short and long-term outcomes after organophosphate poisoning in a rat model. Neurotoxicology 48:206-216

Shubina L, Aliev R, Kitchigina V (2017) Endocannabinoid-dependent protection against kainic acid-induced long-term alteration of brain oscillations in guinea pigs. Brain Res 1661:1-14

Thompson SM, Fortunato C, McKinney RA, Müller M, Gähwiler BH (1996) Mechanisms underlying the neuropathological consequences of epileptic activity in the rat hippocampus in vitro. J Comp Neurol 372:515-528

Wang W, Jia Y, Pham DT, Palmer LC, Jung KM, Cox CD, Rumbaugh G, Piomelli D, Gall CM, Lynch G (2017) Atypical endocannabinoid signaling initiates a new form of memory-related plasticity at a cortical input to hippocampus. Cereb Cortex 17:1-14. doi:10.1093/ cercor/bhx126

Woo JA, Zhao X, Khan H, Penn C, Wang X, Joly-Amado A, Weeber E, Morgan D, Kang DE (2015) Slingshot-Cofilin activation mediates mitochondrial and synaptic dysfunction via $A \beta$ ligation to $\beta 1$ integrin conformers. Cell Death Differ 22:921-934 